\title{
Ground-state properties of hard core bosons in one-dimensional harmonic traps
}

\author{
T. Papenbrock \\ Physics Division, Oak Ridge National Laboratory, Oak Ridge, TN 37831, USA
}

(Dated: November 11, 2018)

\begin{abstract}
The one-particle density matrices for hard core bosons in a one-dimensional harmonic trap are computed numerically for systems with up to 160 bosons. Diagonalization of the density matrix shows that the many-body ground state is not Bose-Einstein condensed. The ground state occupation, the amplitude of the lowest natural orbital, and the zero momentum peak height scale as powers of the particle number, and the corresponding exponents are related to each other. Close to its diagonal, the density matrix for hard core bosons is similar to the one of noninteracting fermions.

PACS numbers: 03.75.Fi, 05.30.Jp, 03.65.Ge
\end{abstract}

Bose-Einstein condensation (BEC) in dilute atomic vapors is a very attractive field of contemporary scientific research. Of particular interest are one-dimensional systems [1] which are experimentally realized in very elongated three-dimensional traps [2]. The one-dimensional Bose gas displays a rich behavior ranging from the weakly interacting regime [3] to the experimentally realized Thomas-Fermi regime to a gas of impenetrable hard core bosons [4, 旬, 6] at sufficiently low densities and large $s$-wave scattering length. This latter case of hard core interactions is particularly interesting since the exact many-body ground-state wave function [See Eq. (1)] is known due to a boson-fermion mapping [7]. In spite of this knowledge, it is nontrivial to extract the groundstate properties from this wave function. The main difficulty to overcome is the computation of the one-particle density matrix for sufficiently large systems. Girardeau et al. [6] used Monte Carlo integration techniques to compute the density matrix for small systems with up to $N=10$ bosons. More accurate methods employed by Lapeyre et al. [8] are restricted to up to $N=8$ particles. Analytical results were derived by Kolomeisky et al. [9] for the diagonal of the density matrix and by Minguzzi et al. 10] for the tails of the momentum distribution. It is the purpose of this work to compute the one particle density matrix for large systems containing more than a hundred particles and to present analytical results and scaling relations as well.

The ground-state wave function for $N$ hard core bosons is given by

$$
\psi_{B}\left(z_{1}, \ldots, z_{N}\right)=C_{N}^{\frac{1}{2}} \prod_{k=1}^{N} e^{-z_{k}^{2} / 2} \prod_{1 \leq i<j \leq N}\left|z_{i}-z_{j}\right|
$$

where the normalization constant is

$$
C_{N}=2^{N(N-1) / 2} \pi^{-N / 2}\left(\prod_{n=1}^{N} n !\right)^{-1} .
$$

Note that the wave function (1) is simply the absolute value of the ground-state wave function $\psi_{F}$ for $N$ noninteracting fermions [7], i.e. $\psi_{B}=\left|\psi_{F}\right|$. The one-particle density matrix is defined as

$$
\begin{aligned}
& \rho_{B}(x, y)=N \int d z_{1} \ldots d z_{N-1} \psi_{B}^{*}\left(z_{1}, \ldots, z_{N-1}, x\right) \\
& \times \psi_{B}\left(z_{1}, \ldots, z_{N-1}, y\right)
\end{aligned}
$$

and requires the integration over $N-1$ variables. Let us rewrite the one-particle density matrix as

$$
\begin{aligned}
\rho_{B}(x, y)= & \frac{2^{N-1}}{\pi^{1 / 2}(N-1) !} \exp \left\{-\frac{1}{2}\left(x^{2}+y^{2}\right)\right\} \\
& \times \int d \mu_{N-1} \prod_{k=1}^{N-1}\left|z_{k}-x\right|\left|z_{k}-y\right| .
\end{aligned}
$$

Here we introduce the integration measure

$$
d \mu_{N-1} \equiv C_{N-1} \prod_{k=1}^{N-1} d z_{k} e^{-z_{k}^{2}} \prod_{1 \leq i<j \leq N-1}\left(z_{i}-z_{j}\right)^{2},
$$

which is identical to the joint probability density for eigenvalues $z_{1}, \ldots, z_{N-1}$ of $(N-1)$-dimensional random matrices drawn from the Gaussian Unitary Ensemble (GUE) [11, 12].

This observation greatly facilitates the Monte Carlo integration since it is easy to generate independent configurations $z_{1}, \ldots, z_{N-1}$ that are distributed according to the measure (5). In practice, one draws a $(N-1)$-dimensional random matrix from the GUE, i.e. the matrix is complex Hermitian, and its matrix elements are Gaussian random variables with zero mean and variance $1 / 2$. Upon diagonalization, one obtains eigenvalues $z_{1}, \ldots, z_{N-1}$ that are distributed according to the measure (5). The integrand $\prod_{k=1}^{N-1}\left|z_{k}-x \| z_{k}-y\right|$ is then evaluated for this configuration, and the procedure is repeated many times. This procedure requires an effort of $O\left(N^{3}\right)$ for each configuration. The computation of the density matrix for $N=10$ bosons takes about one hour on a PC and requires $10^{6}$ configurations. While this is already a considerable improvement over previous integration techniques [6, 8], it is not sufficient when much larger systems are considered.

Following Mehta [1], we express the measure ([D) in 
terms of harmonic oscillator wave functions $\varphi_{n}(z)$ as

$$
d \mu_{N-1}=\frac{d z_{1} \ldots d z_{N-1}}{(N-1) !}\left(\operatorname{det}\left[\varphi_{k-1}\left(z_{l}\right)\right]_{k, l=1, \ldots, N-1}\right)^{2}
$$

The integration can be performed since the $(N-1)$ dimensional integral factorizes

$$
\begin{aligned}
& \int d z_{1} \ldots d z_{N-1}\left(\operatorname{det}\left[\varphi_{k-1}\left(z_{l}\right)\left|z_{l}-x\right|\right]_{k, l=1, \ldots, N-1}\right) \\
& \quad \times\left(\operatorname{det}\left[\varphi_{i-1}\left(z_{j}\right)\left|z_{j}-y\right|\right]_{i, j=1, \ldots, N-1}\right) \\
& \quad=(N-1) ! \operatorname{det}\left[B_{m, n}(x, y)\right]_{m, n=0, \ldots, N-2}
\end{aligned}
$$

Here we introduce the $(N-1)$-dimensional square matrix with elements

$$
B_{m, n}(x, y) \equiv \int_{-\infty}^{\infty} d z|z-x||z-y| \varphi_{m}(z) \varphi_{n}(z) .
$$

We may thus express the one-particle density matrix of $N$ bosons as the determinant of a $(N-1)$-dimensional matrix

$\rho_{B}(x, y)=\frac{2^{N-1} e^{-\left(x^{2}+y^{2}\right) / 2}}{\pi^{1 / 2}(N-1) !} \operatorname{det}\left[B_{m, n}(x, y)\right]_{m, n=0, \ldots, N-2}$.

This form of the density matrix and its connection to the GUE was previously discussed by Forrester et al. 113. The density matrix $\rho_{B}(x, y)$ may now be computed numerically. For a numerical computation of Hermite polynomials, see, e.g., Refs. 114, 15]. The total effort scales like $O\left(N^{5}\right)$, and the calculation for a system of $N=100$ bosons requires about one day on a PC. We computed the density matrix for systems containing up to $N=160$ particles. Figures 1 and 2 show density plots of the density matrix $\rho_{B}(x, y)$ for $N=10$ and $N=100$ particles, respectively. The salient features are strong intensities close to the diagonal $x=y$ and small off-diagonal contributions which rapidly drop to zero at $|x|,|y| \approx R$, where

$$
R \equiv(2 N)^{1 / 2}
$$

is the radius of the density $\rho_{B}(x, x)$ [9] or Wigner's semicircle [11. Note that the width of the peak along the diagonal decreases with increasing particle number.

Diagonalization of the density matrix yields the natural orbitals $\phi_{j}(x)$ and occupation numbers $n_{0} \geq n_{1} \geq$ $n_{2} \geq \ldots \geq 0$ fulfilling $\int d y \rho_{B}(x, y) \phi_{j}(y)=n_{j} \phi_{j}(x)$, i.e.

$$
\rho_{B}(x, y)=\sum_{j} n_{j} \phi_{j}(x) \phi_{j}(y) .
$$

The many-body ground-state is Bose-Einstein condensed provided the density matrix exhibits a macroscopic eigenvalue $n_{0} \propto N$; the corresponding natural orbital $\phi_{0}(x)$ is then the wave function of the BEC. Our data show

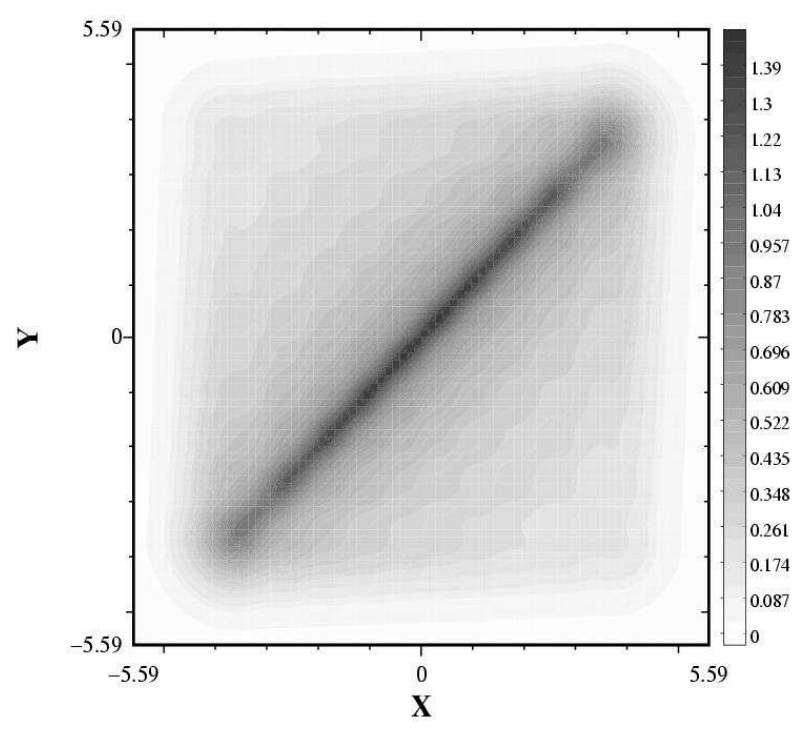

FIG. 1: One-particle density matrix $\rho_{B}(x, y)$ for $N=10$ bosons. Lightest gray indicates almost zero amplitude, while black indicates the maximal amplitude $\approx(2 N)^{1 / 2} / \pi$. The width of the diagonal peak is proportional to $N^{-1 / 2}$.

that $n_{0}$ is not simply a power of $N$ for the limited range of particle numbers considered in this work. Previous studies on small systems suggested that $n_{0} \propto N^{\alpha}$ with $\alpha \approx 0.59$ [6]. While this value is a good fit for systems with up to $N=10$ particles, we find that the exponent $\alpha$ actually decreases with increasing particle number $N$, reaching $\alpha \approx 0.53$ for the largest particle numbers considered in this work. Below we will present simple scaling arguments which suggest that $\alpha=0.5$ is the expected behavior for large particle number $N$. This behavior of harmonically trapped hard core bosons is similar to the uniform system of hard core bosons [16].

Figure 3 shows the scaled natural orbitals $R^{1 / 2} \phi_{0}(x)$ for $N=10,40,160$ bosons. Note that the scaled natural orbitals approach an $N$-independent function that depends only on the scaled variable $x / R$ as $N$ increases. This function is nonzero only for $|x| / R \lesssim 1$ and varies smoothly over this interval. We find that the maximum amplitude of the lowest natural orbital scales like $\phi_{0}(0) \propto N^{\beta}$ with $\beta=-0.25$. This is expected since the natural orbital $\phi_{0}$ is normalized and is supported on a domain that scales like $R \propto N^{1 / 2}$.

The momentum distribution

$$
n(k) \equiv \frac{1}{2 \pi} \int d x d y \rho_{B}(x, y) e^{-i k(x-y)}
$$

is of particular interest. Plots of the normalized momentum distribution $n(k) / N$ are shown in Figure 1 for $N=10,40,160$. The distributions have a pronounced peak at zero momentum and long tails. Minguzzi et al. [10] showed that the tails of the momentum distribution decay as $n(k) \propto k^{-4}$ for large momenta $k$. We find that 


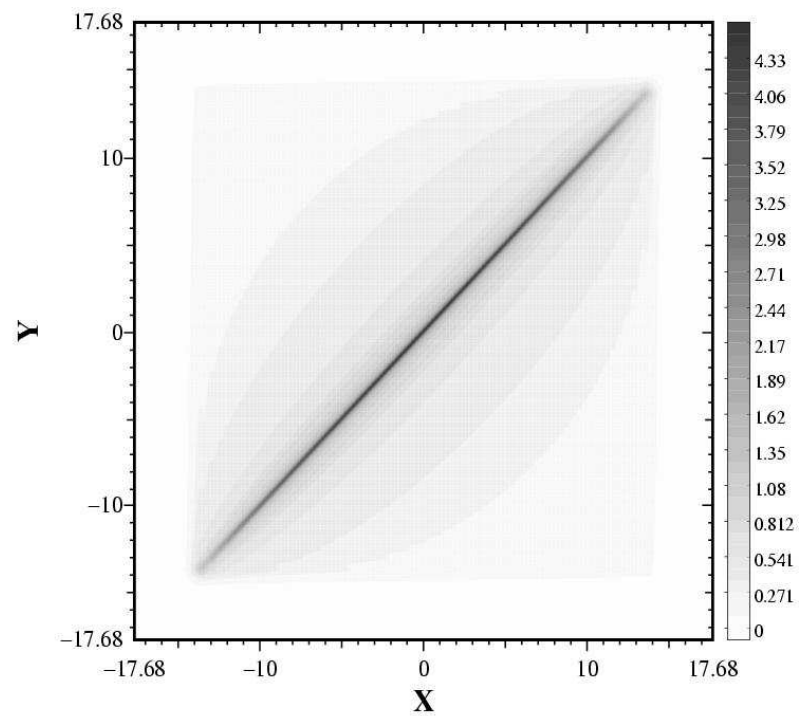

FIG. 2: Same as Figure 1, but for $N=100$ particles.

the peak height $n(0)$ is proportional to the particle number, $n(0) \propto N^{\gamma}$ with $\gamma=1.0$ (see the inset of Figure 4). Thus, the system of hard core bosons mimics the macroscopic occupation of a momentum zero state and in this aspect resembles a uniform and noninteracting Bose system.

This finding is particularly interesting, because it allows us to predict the $N$-dependence of the ground-state occupation $n_{0}$. According to Eq. (12), the momentum peak height $n(0)$ is simply the integral over the density matrix which we approximate as

$$
\begin{aligned}
n(0) & \propto \int d x d y \rho_{B}(x, y) \\
& \text { diagonal region } \\
& +n_{0} \int d x d y \phi_{0}(x) \phi_{0}(y) . \\
& \text { off-diagonal domain }
\end{aligned}
$$

Here we have decomposed the domain of integration into the diagonal region over the peaked structure and the offdiagonal contribution; the latter may be approximated in leading order by the integral over the lowest natural orbital $\phi_{0}$ since all other natural orbitals have smaller occupation number and their positive and negative amplitudes lead to cancellations when integrated over the off-diagonal domain. The contribution from diagonal region scales like $N^{1 / 2}$ and can therefore be neglected when compared with the momentum peak $n(0) \propto N^{1.0}$. This can be seen as follows. The normalized density along the diagonal is proportional to $N$, while the width of the diagonal peak decreases like $N^{-1 / 2}$ with increasing particle number (see below). The remaining off-diagonal contribution scales like $n_{0} R^{2}\left(\phi_{0}(0)\right)^{2}=n_{0} N^{0.5}$ since the domain of integration is a square whose area scales like $R^{2} \propto N$. Thus, $n_{0} \propto N^{\alpha}$ with $\alpha=0.5$. Let us summa-

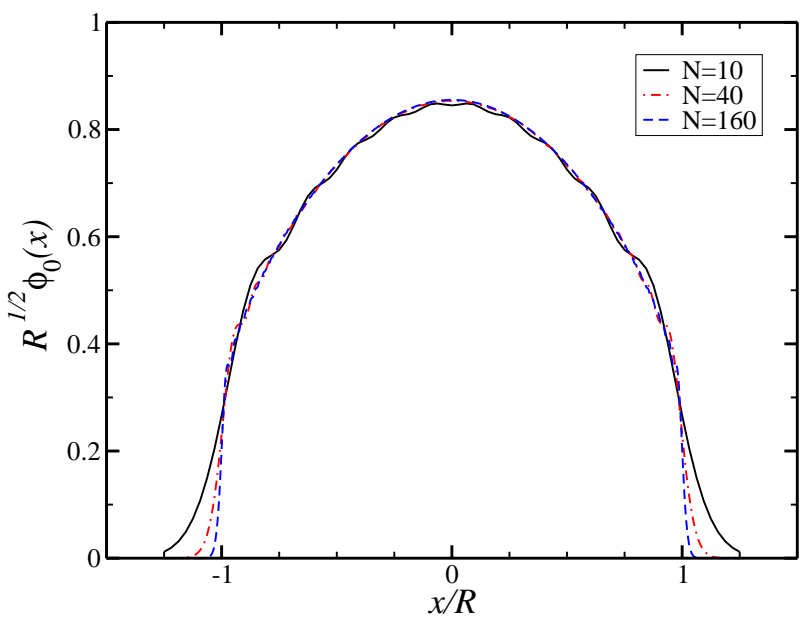

FIG. 3: Scaled natural orbital $R^{1 / 2} \phi_{0}(x)$ for $N=10$ (full line), $N=40$ (dashed-dotted line), and $N=160$ (dashed line) bosons. $R=(2 N)^{1 / 2}$ sets the length scale in units of the oscillator length. The natural orbitals $\phi_{0}(x)$ are nonzero on an interval that scales like $N^{1 / 2}$ and have a maximum amplitude proportional to $N^{-0.25}$.

rize the analysis of this paragraph into the equation

$$
\gamma=1+\alpha+2 \beta
$$

that relates the scaling of the momentum peak height to the ground state occupation and the amplitude of the lowest natural orbital.

The density matrix $\rho_{B}(x, y)$ can be understood analytically close to its diagonal $x=y$. Kolomeisky et al. [9] showed that the diagonal density matrix is identical to the density of noninteracting fermions in one-dimensional harmonic traps, i. e. $\rho_{B}(x, x)=\sum_{n=0}^{N-1} \varphi(x) \varphi(x)$, which is also identical to the level density of GUE random matrices 11. This analogy holds also in leading order as one leaves the diagonal. To see this, we write the matrix (8) as

$B_{m, n}(x, y)=F_{m, n}-2 \int_{\min (x, y)}^{\max (x, y)} d z(z-x)(z-y) \varphi_{m}(z) \varphi_{n}(z)$

with

$$
\begin{aligned}
& F_{m, n} \equiv \int_{-\infty}^{\infty} d z(z-x)(z-y) \varphi_{m}(z) \varphi_{n}(z) \\
& =\left(x y+n+\frac{1}{2}\right) \delta_{m}^{n}-\frac{x+y}{\sqrt{2}}\left(\sqrt{n} \delta_{m}^{n-1}+\sqrt{m} \delta_{m-1}^{n}\right) \\
& +\frac{1}{2}\left(\sqrt{m(n+1)} \delta_{m-1}^{n+1}+\sqrt{n(m+1)} \delta_{m+1}^{n-1}\right) .
\end{aligned}
$$

The decomposition of the matrix elements $B_{m, n}$ into the matrix elements $F_{m, n}$ and a remainder is quite useful. The remaining integral in Eq. (15) vanishes on the diagonal $x=y$ and yields corrections proportional to $|x-y|^{3}$ 


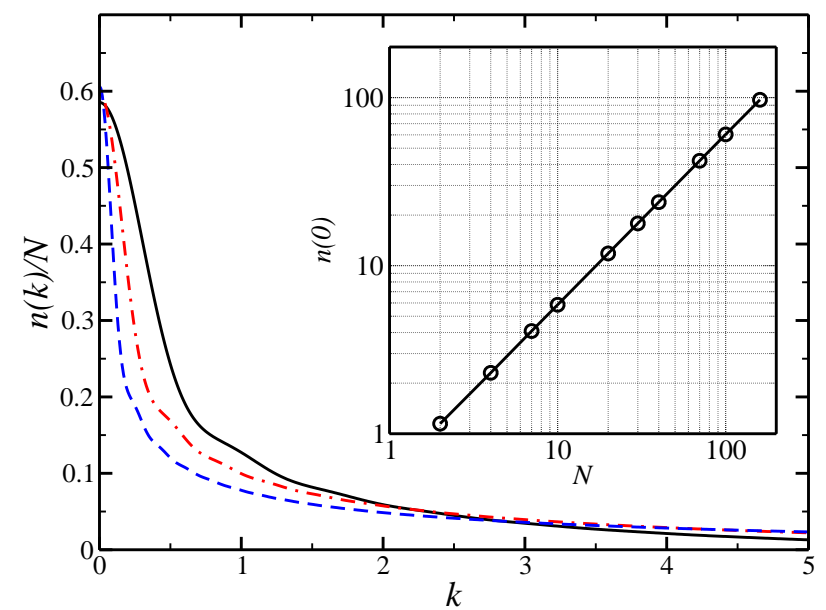

FIG. 4: Normalized momentum distribution $n(k) / N$ for systems of $N=10$ (full line), $N=40$ (dashed-dotted line), and $N=160$ (dashed line) bosons. The momentum $k$ is given in units of the inverse oscillator length. The inset shows the zero-momentum peak $n(0)$ as a function of particle number $N$ in a log-log plot.

as one leaves the diagonal. The leading corrections proportional to $(x-y)^{2}$ are already contained in the matrix elements $F_{m, n}$. Thus, close to the diagonal, the matrix $B$ can be approximated by the matrix $F$. This latter matrix, however, determines the density matrix $\rho_{F}(x, y)$ for noninteracting fermions in a one-dimensional harmonic trap. Indeed, adjusting the above calculation to the case of noninteracting fermions yields

$\rho_{F}(x, y)=\frac{2^{N-1} e^{-\left(x^{2}+y^{2}\right) / 2}}{\pi^{1 / 2}(N-1) !} \operatorname{det}\left[F_{m, n}(x, y)\right]_{m, n=0, \ldots, N-2}$

for the fermionic density matrix. [Note that the more familiar expression $\rho_{F}(x, y)=\sum_{n=0}^{N-1} \varphi_{n}(x) \varphi_{n}(y)$ for the same density matrix can be obtained in a direct calculation]. Thus, the density matrix for hard core bosons is practically identical to the density matrix for noninteracting fermions close to its diagonal. Note, finally, that the square of the fermionic density matrix $\rho_{F}^{2}(x, y)$ is identical to the two-level cluster function of the GUE [11. This allows us to transfer results obtained for random matrices to the case of noninteracting fermions. Close to the origin, we thus find for large particle number $N \gg 1$ and $\delta \ll N^{-1 / 2}$

$$
\rho_{B}(\delta,-\delta) \approx \rho_{F}(\delta,-\delta)=\frac{1}{\pi} \sqrt{2 N}\left(1-\frac{4}{3} N \delta^{2}\right) .
$$

Thus, the width and height of the prominent peak along the diagonal of the bosonic density matrix (see, e.g., Figures [1, 2) scales like $N^{-1 / 2}$ and like $N^{1 / 2}$, respectively. This behavior is confirmed by our numerical computations where we take the width at half maximum.
In summary, we expressed the density matrix of $N$ harmonically trapped hard core bosons as a determinant of a $(N-1)$-dimensional symmetric matrix and performed numerical computations on systems containing more than a hundred particles. The density matrix is strongly peaked along its diagonal but lacks off-diagonal long range order. Accordingly, the ground-state is not Bose-Einstein condensed. The ground-state occupation, the amplitude of the lowest natural orbital, and the momentum peak height scale as powers of the particle number, and the corresponding exponents are related to each other by a simple equation. The lowest natural orbital approaches a particle number independent function when scaled properly. Analytical results show that the density matrices of hard core boson systems and noninteracting fermion systems are almost identical close to the diagonal.

The author acknowledges communications with P. J. Forrester. This research used resources of the Center for Computational Sciences at Oak Ridge National Laboratory (ORNL). ORNL is managed by UT-Battelle, LLC for the U.S. Department of Energy under contract DEAC05-00OR22725.

[1] W. Ketterle and N. J. van Druten, Phys. Rev. A 54, 656 (1996).

[2] A. Görlitz, J. M. Vogels, A. E. Leanhardt, C. Raman, T. L. Gustavson, J. R. Abo-Shaeer, A. P. Chikkatur, S. Gupta, S. Inouye, T. P. Rosenband, D. E. Pritchard, and W. Ketterle, Phys. Rev. Lett. 87, 130402 (2001).

[3] T. Papenbrock, Phys. Rev. A 65, 033606 (2002).

[4] D. S. Petrov, G. V. Shlyapnikov, and J. T. M. Walraven, Phys. Rev. Lett. 85, 3745 (2000).

[5] V. Dunjko, V. Lorent, and M. Olshanii, Phys. Rev. Lett. 86, 5413 (2001).

[6] M. D. Girardeau, E. M. Wright, and J. M. Triscari, Phys. Rev. A 63, 033601 (2001).

[7] M. D. Girardeau, Phys. Rev. 139, B500 (1965).

[8] G. J. Lapeyre, Jr., M. D. Girardeau, and E. M. Wright, Phys. Rev. A 66, 023606 (2002).

[9] E. B. Kolomeisky, T. J. Newman, J. P. Straley, and Xiaoya Qi, Phys. Rev. Lett. 85, 1146 (2000).

[10] A. Minguzzi, P. Vignolo, and M. P. Tosi, Phys. Lett. A 294, 222 (2002).

[11] M. L. Mehta, Random Matrices (Academic, New York, 1991), 2nd ed.

[12] T. Guhr, A. Müller-Groeling, and H. A. Weidenmüller, Phys. Rep. 299, 189 (1998).

[13] P. J. Forrester, N. E. Frankel, T. M. Garoni, and N. S. Witte, math-ph/0207005.

[14] S. Zhang and J. M. Jin, Computation of Special Functions (New York, John Wiley \& Sons, 1996).

[15] W. Gautschi, ACM Transactions on Mathematical Software 20, 21 (1994).

[16] A. Lenard, J. Math. Phys. 7, 1268 (1966). 